\title{
Ellagic acid inhibited cervical cancer growth via blocking the AKT/mTOR/STAT3 pathway
}

Jinyu Xia ${ }^{1}$, Chunyu Xue ${ }^{2}$, Jie Yu ${ }^{1}$

${ }^{1}$ Department of Gynecology, The Affiliated Hospital of Qingdao University, Qingdao, Shandong, China

2Department of Emergency, Qingdao Municipal Hospital, Qingdao, Shandong, China

Submitted: 3 August 2020

Accepted: 17 September 2020

Arch Med Sci

DOI: https://doi.org/10.5114/aoms.2020.100837

Copyright $\odot 2020$ Termedia \& Banach

\section{Abstract}

Introduction: Ellagic acid (EA) is a kind of herb extract. However, the effects and mechanisms of EA in cervical cancer treatment are unclear.

Material and methods: Our present work investigated the effects of EA on HeLa cervical cancer cell biological activities and relative mechanisms. Hela cells were divided into 5 groups as the NC group, Low group, Middle group, High group and 5-Fu group. MTT assay and cell apoptosis assay were performed to evaluate cell proliferation and apoptosis. Wound closure assay was used to assess the effect of EA on HeLa cell migration. We also assessed the anti-tumor effect of EA on a cervical tumor bearing BALB/c mouse model. Furthermore, western blotting and immunohistochemistry assay were performed to evaluate the effect of EA on the activation of AKT/mTOR/STAT3 in vitro and in vivo.

Results: The cell experiments showed that EA had effects to inhibit Hela cell biological activities including cell apoptosis, migration and invasion dose-dependently and AKT, mTOR, and STAT3 phosphorylation expression levels were significantly depressed after EA supplementation in the in vitro study. In the in vivo study, EA significantly depressed tumor growth with cell apoptosis significantly increasing and the AKT/mTOR/STAT3 pathway was significantly down-regulated in tumor tissues.

Conclusions: EA had anti-tumor effects on cervical cancer by depressing the AKT/mTOR/STAT3 pathway in in vitro and in vivo study.

Key words: ellagic acid, AKT, mTOR, HeLa cervical cancer cells, cervical tumor.

\section{Introduction}

Phenolic compounds are among the important secondary metabolites in fruits and vegetables. Their beneficial effects on human health are related to antioxidant and anti-inflammatory effects and potential tumor prevention [1]. Relevant studies have confirmed that eating vegetables and fruits rich in polyphenols has a positive significance for the prevention of various tumors [2]. Ellagic acid (EA) is a kind of polyphenol existing in a variety of natural foods. It is considered as a candidate compound for clinical application because of its antioxidant capacity and potential antitumor effect [3-5]. EA showed its antitumor activity by eliminating MDR, inducing apoptosis and caspase-3 expression [6-9].

\author{
Corresponding author: \\ Jie Yu \\ Department \\ of Gynecology \\ The Affiliated Hospital \\ of Qingdao University \\ Qingdao, Shandong \\ 266003, China \\ E-mail: yuss109@163.com
}


Cervical cancer and cervical precancerous lesions are still a global health problem. Despite various screening and treatment methods, cervical cancer is still the third leading cancer in the world and the main cause of cancer in women [10]. Cervical cancer is closely related to human papillomavirus (HPV) infection [11]. HPV cancer protein E6 interacts with cell protein E6AP, which is a ubiquitin enzyme that catalyzes the ubiquitination of p53 and leads to the degradation of tumor suppressor protein [12]. Cancer involves many signaling pathways. Therefore, the treatment should include molecules that target specific signaling pathways [13].

It is worth noting that the signal transduction and activator of transcription (STAT3) of the mammalian target of serine threonine protein kinase (Akt)/rapamycin (mTOR) has been proved to be essential for the survival and proliferation of cancer cells [14]. STAT3 is continuously activated by non-receptor tyrosine kinases such as Akt and mTOR. The Akt/mTOR pathway has been widely reported as an effective signal axis to promote survival and metastasis, while new drugs specifically inhibiting its activation provide a new targeted treatment for many cancers [15]. STAT3 activation can lead to the enhancement of oncogene expression [16].

In previous reports on the role of EA and tumor cells, researchers mainly used cancer cells, such as colon, prostate, oral, pancreatic cells and the leukemia cell line HL-60 [1, 7, 17-19]. EA extracted from pomegranate has antitumor activity towards a variety of cancer cells. Electroacupuncture induces G0/G1 arrest and apoptosis of human cervical cancer CaSki cells [1]. Researchers are interested in natural compounds that enhance cancer prevention and treatment. In this study, the Akt/mTOR/STAT3 axis was used as the target to observe the anti-cancer effect of electroacupuncture on HeLa cervical cancer cells, and to observe the dose-dependent induction of apoptosis, inhibition of cell proliferation, migration and anti-tumor growth activity of electroacupuncture.

\section{Material and methods}

\section{Reagents}

The HeLa cell line of human cervical cancer was obtained from NCCS in Pune, India. Dulbecco's modified Eagle medium (DMEM) was obtained from MediaTek in Herndon, Virginia. Fetal bovine serum (FBS), penicillin/Streptomycin were obtained from GIBCO. Ellagic acid (EA) and staurosporine were purchased from Sigma Aldrich (St. Louis, Mo). Ellagic acid was diluted in $0.1 \mathrm{M} \mathrm{NaOH}$ and sterile filtered through a $0.2 \mathrm{M}$ nylon filter
(SMI Labhut, Gloucester, UK). Rabbit anti-Akt polyclonal antibody (SC-8312), rabbit anti-P-AKT polyclonal antibody (SC-7985) and mouse anti-p-PI3K monoclonal antibody (sc-12929), rabbit anti-Stat3 polyclonal antibody (sc-7179), rabbit anti-p-Stat3 polyclonal antibody (sc-135649), and mouse anti-EAPDH monoclonal antibody (sc-32233) were from Santa Cruz Biotechnology (Santa Cruz, CA, USA). Mouse anti-PI3K polyclonal antibody (ab86714), rabbit anti-mTOR polyclonal antibody (ab45989) and rabbit anti-pmTOR polyclonal antibody (ab2732) were from Abcam (BurlinEAme, CA USA). (3-(4,5)-dimethylthiazol-2-yl)-2,5-diphenyltetrazolium bromide (MTT) was from Sigma Aldrich (St Louis, MO, USA). The TACSH annexin V (AnV) fluorescein isothiocyanate (FITC) kit (4830-01-K) was from Trevigen (EAL, MD, USA).

\section{Cell culture and treatment}

HeLa cells were cultured in DMEM medium with $10 \%$ fetal bovine serum, $100 \mathrm{U} / \mathrm{ml}$ penicillin and $100 \mathrm{U} / \mathrm{ml}$ streptomycin (pH 7.2). Cells were cultured in a $5 \% \mathrm{CO}_{2}$ humidified incubator at $37^{\circ} \mathrm{C}$. The cells grew to about $70-80 \%$ and then transferred to a $10 \mathrm{~cm}$ culture dish for further culture. HeLa cells were treated with different concentrations $(0 \mu \mathrm{M}, 2.5 \mu \mathrm{M}, 5 \mu \mathrm{M}, 10 \mu \mathrm{M} ; 0 \mu \mathrm{M}$ as control) of EA and 5 -Fu for $48 \mathrm{~h}$ at $37^{\circ} \mathrm{C}, 5 \% \mathrm{CO}_{2}$.

\section{Western blot analysis}

After treatment, cells were rinsed with cold phosphate buffer. On ice, they were dissolved in RIPA buffer (50 mm Tris, pH 7.2; $1 \%$ sodium deoxycholate; $150 \mathrm{~mm} \mathrm{NaCl}$; $0.1 \%$ sodium dodecyl sulfate; $10 \mathrm{~mm} \mathrm{NaF;} 1 \%$ Triton X100; $1 \mathrm{~mm}$ $\mathrm{Na}_{3} \mathrm{VO}_{4}$; protease inhibitor mixture $\left.(1: 1000)\right)$. The lysate was treated with ultrasound for $10 \mathrm{~s}$, centrifuged at $4^{\circ} \mathrm{C}$ for $13000 \mathrm{~g}$ for $10 \mathrm{~min}$, and the concentration of BSA was determined by the method of dioctanoic acid. The same amount of protein was separated on the $7.5-12 \%$ twelve alkyl sulfate polyacrylamide gel and transferred onto the PVDF membrane. The membrane was incubated with PBS containing $0.05 \%$ Tween $20 \%$ and $5 \%$ skimmed milk powder to prevent nonspecific binding, and then incubated with primary antibody, and then combined with appropriate secondary antibody to produce horseradish peroxidase. The band density was quantitatively analyzed with the density measurement software Gel-Pro Analyzer (media control netics, MD, USA).

\section{QRT-PCR}

According to the manufacturer's instructions, total RNA was extracted from cultured cells using TRIzol reagent (Invitrogen, Carlsbad, CA, USA). The first strand of CDNA was generated using Prime- 
Script Gamma RT Kit (Takara, Japan). We used SYBR premier ex Taq for quantitative real-time PCR Gamma II (Japanese plateau). GAPDH is the internal control of mRNA expression. The primer sequence was designed according to the requirements.

\section{Cell proliferation assay}

The 3-(4,5-dimethylthiazol-2-yl)-2,5-diphenyltetrazolium bromide (MTT) method was used to determine the inhibitory effect of EA on HeLa cell proliferation. Simply put, the cells were inoculated on 96-well plates for $12 \mathrm{~h}$, treated with different concentrations of EA for $72 \mathrm{~h}$, then $50 \mathrm{ml}$ of MTT solution (DMEM w/O phenol red $5 \mathrm{mg} / \mathrm{ml}$ ) was added to each well, further incubated for 4 $\mathrm{h}$, the culture medium was absorbed, $80 \mathrm{ml}$ of DMSO was added to dissolve the purple formalin crystal, and slowly shaken in a shaker for $30 \mathrm{~min}$. The absorbance was measured at $570 \mathrm{~nm}$ with a microplate reader. The cell proliferation rate was calculated.

\section{Transwell assay}

The Hela cells were seeded on the surface of carbonate phospholipid Transwell chambers, the upper chamber Matrigel coated with BioCoat (BD Biosciences, San Jose, CA). The Hela cells were cultured at $37^{\circ} \mathrm{C}$ for $24 \mathrm{~h}$. Remove the upper chamber, the lower chamber of the cell invasion and $1 \%$ paraformaldehyde mixed with $0.2 \%(\mathrm{w} / \mathrm{w})$ crystal violet staining solution for $15 \mathrm{~min}$. We counted the number of cells entering the membrane with a microscope, randomly took 10 visual fields, and took the average.

\section{Cell migration assay}

HeLa cells were plated in 6-well plates and grown until confluency. A scratch was made in the confluent monolayer using a $200 \mu \mathrm{l}$ pipet tip. The medium was replaced with serum-free DMEM cultured for $48 \mathrm{~h}$.

\section{Cell apoptosis and cell cycle assay}

HeLa cells were treated with EA and 5-FU of different concentrations $(0 \mu \mathrm{m}, 2.5 \mu \mathrm{m}, 5 \mu \mathrm{m}$, $10 \mu \mathrm{m} ; 0 \mu \mathrm{m}$ as control) for $48 \mathrm{~h}$. Cells were collected, washed with cold PBS solution three times, fixed with $70 \%$ ethanol for 30 min, washed with PBS solution three times according to the manufacturer's instructions, and then labeled with annexin V-FITC and disodium iodide (PI) (CST, USA). Flow cytometry (BD Biosciences) was used to analyze the cells. The content of DNA was detected by PI staining and flow cytometry. The experiment was repeated three times.

\section{Xenograft models and \\ immunohistochemistry detections}

Female BALB/c mice (7-8 weeks old) were purchased from NBRI. The animal use procedure was approved by the institutional Committee of the Beckman Institute of Hope Medical Center. Mice were implanted with $2.5 \times 106$ HeLa cervical cancer cells. Mice with appropriate tumor size were randomly divided into 5 groups: control group, low dose group (50 mg/kg/day), middle dose group (75 mg/kg/day), high dose group (100 mg/kg/day) and 5-FU group. Mice were intraperitoneally injected with electroacupuncture. The tumor volume and the weight of mice were measured every 3 days. After the mice were killed on the $30^{\text {th }}$ day, tumor and normal tissues were collected for molecular evaluation. Specifically, tumor and normal tissues were fixed in 10\% PBS solution, dehydrated in $70 \%$ ethanol, embedded in $4 \%$ paraffin at $4^{\circ} \mathrm{C}$, and then sectioned $(5 \mu \mathrm{m})$. The specific antibodies p-pi3k, p-Akt, p-mTOR and p-STAT3 (cell signal transduction) were used to stain the paraffin removed tumor tissue. The compound of avidin biotin horseradish peroxidase (CA) and diaminobiphenylamine (DAP) were used as chromogenic agents. The nuclei were re-stained with hematoxylin and the images were taken under a light microscope.

\section{TUNEL assay}

In order to detect apoptosis in tumor tissue treated by electroacupuncture, the TUNEL test (dUTP nick end labeling mediated by terminal DEOXYNUCLEOTIDYLTRANSFERASE, in situ cell death detection kit, PDO, Roche) was carried out according to the manufacturer's instructions. In short, the tumor tissue sections were dewaxed and infiltrated, washed in PBS, and then incubated for $60 \mathrm{~min}$ in a labeled solution composed of TDT and fluorescein binding deoxynucleotides at $37^{\circ} \mathrm{C}$. After washing with PBS solution three times, the slices were incubated in the solution of transforming POD (anti-fluorescein peroxidase) at $37^{\circ} \mathrm{C}$ for $30 \mathrm{~min}$. We observed them under an optical microscope, took photos and analyzed them with Image J image analysis software.

\section{Statistical analysis}

The data are expressed as mean \pm SD. The differences between the two groups were evaluated by two tailed $t$-test or one-way ANOVA and Dunnett test. The difference was statistically significant at $p<0.05$.

\section{Results}

\section{Ellagic acid inhibits proliferation in vitro}

In order to determine whether electroacupuncture has a direct antitumor effect on HeLa cells, 
the dose-response process of HeLa cells was studied. The MTT method was used to detect the cell activity, and EA significantly inhibited the cell activity in a dose-dependent manner (Figure $1 \mathrm{~A}$ ). Then flow cytometry (FCM) was used to detect apoptosis. Flow cytometry showed that EA could induce apoptosis of HeLa cells in a dose-dependent manner (Figure 1 B).

\section{Ellagic acid induced cell cycle arrest in G2 phase}

We used flow cytometry to detect DNA content of cells to monitor the effect of EA on cell cycle progression. The $\mathrm{G} 1$ phase of any given cell cycle lasts from the end of mitosis ( $M$ phase) to the beginning of DNA synthesis (S phase). Compared with other stages of the cell cycle, EA with G2 phase length changed the distribution of DNA content. At $48 \mathrm{~h}$ after electroacupuncture treatment, the number of cells in $G 2$ phase increased significantly in a dose-dependent manner (Figure $1 \mathrm{C}$ ).

\section{Ellagic acid inhibits cervical cancer cell migration and invasion abilities}

Cell migration and invasion are the key to HeLa cell metastasis. We conducted wound healing and hole experiments to study the effect of electroacupuncture on cell invasion and migration, and observed that electroacupuncture strongly inhibited HeLa cell invasion and migration in a dose-dependent manner (Figures 2 A, B).

\section{Ellagic acid inhibits activation of PI3K/AKT/ mTOR/STAT3 signaling in vitro}

In order to investigate the regulatory mechanism of the effect of EA on HeLa cells, we studied the most important carcinogenic signaling pathway of PI3K/Akt/mTOR/Stat3. Western blot analysis and QRT PCR results showed that EA decreased the levels of phospo-PI3K, phospho-Akt and phospho-mTOR in HeLa cells in a dose-dependent manner (Figures $3 \mathrm{~A}, \mathrm{~B}$ ). Considering that STAT3 is continuously activated in a variety of cancers (including HeLa cells), we evaluated whether EA induced tumor growth and migration inhibition is related to STAT3 inhibition. Our results showed that EA significantly inhibited the expression of activated STAT3 in HeLa cells (Figures 3 A, B).

\section{Ellagic acid inhibited cervical cancer growth in vivo}

Next we evaluated whether electroacupuncture inhibited tumor growth in vivo. Compared with the carrier control group, ellagic acid can significantly inhibit tumor volume, indicating that ellagic acid can significantly inhibit the growth of cervical can- cer (tumor volume and weight) (Figure 4 A). Immunohistochemical TUNEL staining also showed that EA induced apoptosis of HeLa cells (Figure $4 \mathrm{~B})$. In conclusion, these data indicate that EA has a strong antitumor effect on HeLa cells. Electroacupuncture can effectively inhibit the growth of cervical cancer, which is related to the decrease of $p$-PI3k, p-AKT, p-mTOR and p-STAT3 activities in the tumor (Figure 5). At the same time, there was no significant difference in the weight of mice in each group, indicating that electroacupuncture had little toxic effect on mice. In conclusion, these data indicate that EA can significantly reduce the growth of cervical cancer in vivo.

\section{Discussion}

Cervical cancer is a leading cause of death for women all over the world [10]. Although the current chemotherapy is effective in some cases, it cannot be completely cured, and there are serious side effects [20-23]. Therefore, the use of less toxic compounds to kill cancer cells is of great value to alternative methods with lower toxicity towards healthy cells. Some reports show that plant-derived compounds have a killing effect on cervical cancer cells [24, 25]. In this study, we used ellagic acid to characterize their anticancer properties.

The proliferation, migration and invasion of cells are closely related to the progress of tumor, so inhibiting the growth and migration of tumor cells may be an important means to prevent tumor progression [26]. HeLa cell activity enhancement (proliferation, invasion and migration) plays an important role in the occurrence and development of cervical cancer. Therefore, we studied whether EA has antitumor activity towards HeLa cells. The results showed that EA significantly inhibited the proliferation, invasion and migration of HeLa cells. It was also found that the Akt/mTOR/STAT3 signaling pathway was significantly inhibited with EA intervention. In addition, the effect of EA on HeLa cells may be mediated by the Akt/mTOR/STAT3 pathway.

The AKT/mTOR/STAT3 signaling pathway is an important intracellular signaling pathway in tumor cell growth and migration [27]. Phosphorylated AKT and mTOR are transported to the nucleus to transmit extracellular signals regulating cell growth, differentiation, proliferation, apoptosis and migration [28]. STAT3 is considered to be an important regulator of cytoskeleton dynamics, transcription, cell cycle progression and cell transformation [29]. STAT3 activation has been shown to regulate the migration and proliferation of leukemia cells. Therefore, drug targeting of the AKT/ mTOR/STAT3 pathway may be helpful for tumor treatment [30]. In the current study, we found that EA can inactivate the AKT/mTOR/STAT3 pathway. 

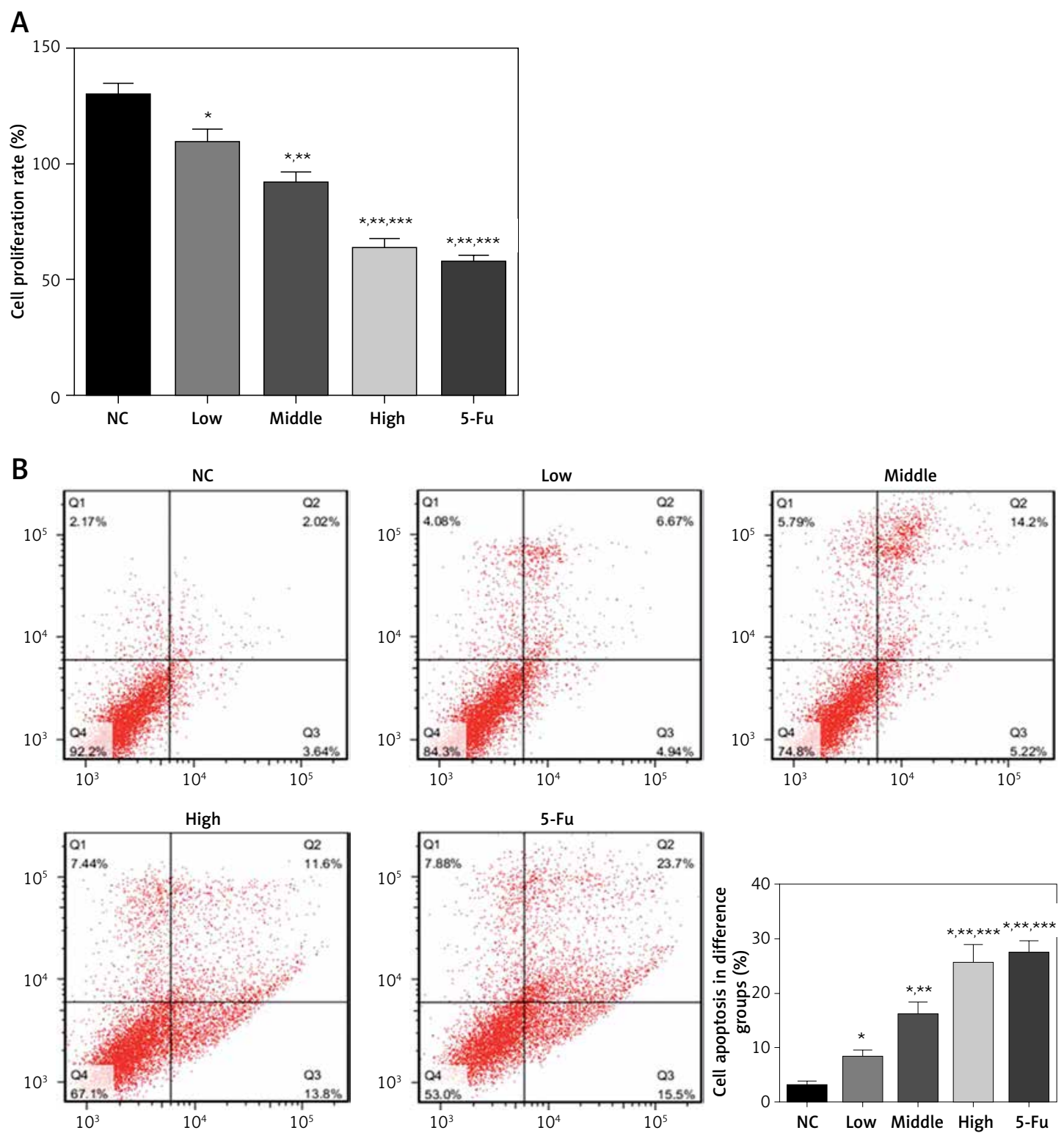

Figure 1. Effect of EA on HeLa cell proliferation and apoptosis. A - MTT assay was performed for cell proliferation detection. B - HeLa cell apoptosis was measured by Annexin V-FITC apoptosis detection kit: NC - cells treated with $0 \mu \mathrm{M}$ EA. Low - cells treated with $2.5 \mu \mathrm{M}$ EA. Middle - cells treated with $5 \mu \mathrm{M}$ EA. High - cells treated with $10 \mu \mathrm{M}$ EA. 5-Fu - cells treated with appropriate concentration of 5-Fu 


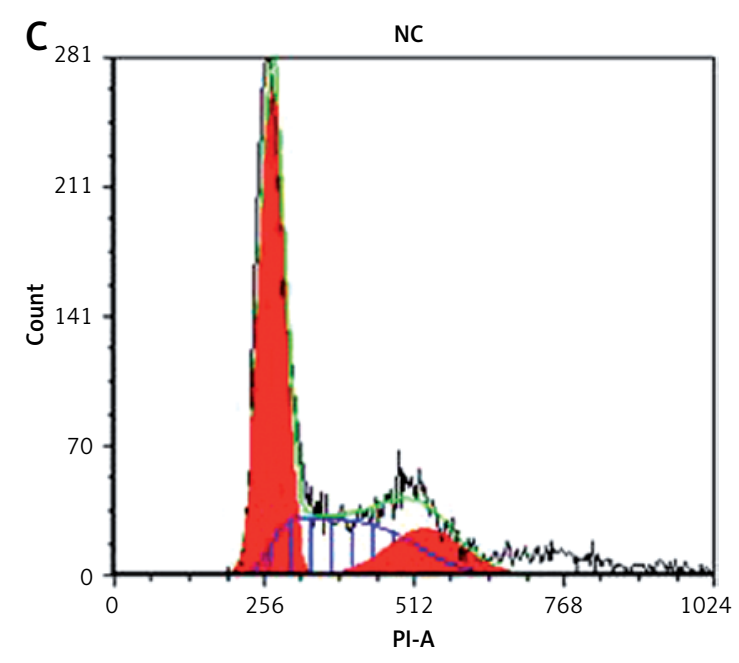

\begin{tabular}{|c|c|c|c|c|c|c|c|c|c|c|}
\hline Cycle & G1 Mean & G1 CV & \%G1 & G2 Mean & G2 CV & \%G2 & \%S & G2/G1 & \%Total & B.A.D. \\
\hline Diploid & 270.45 & 7.76 & 53.74 & 532.77 & 12.01 & 15.60 & 30.67 & 1.97 & 100.00 & 0.53 \\
\hline
\end{tabular}

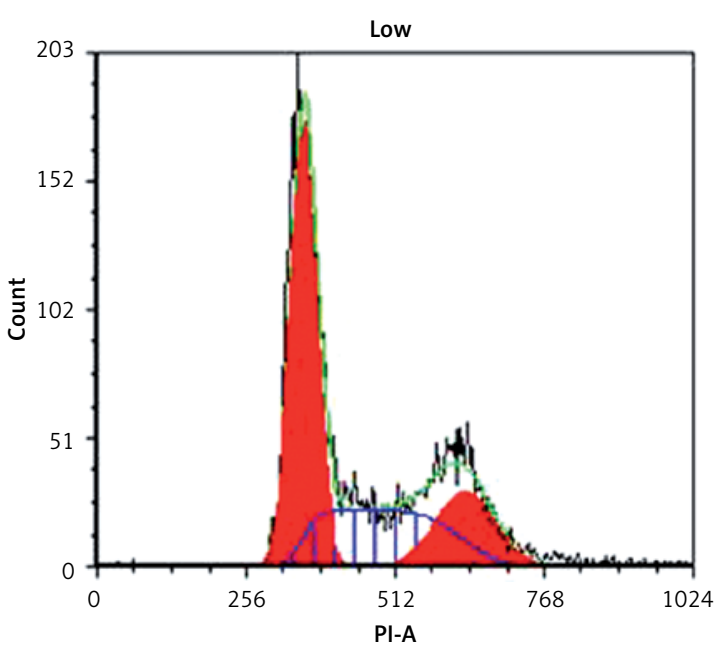

\begin{tabular}{|c|c|c|c|c|c|c|c|c|c|c|}
\hline Cycle & G1 Mean & G1 CV & \%G1 & G2 Mean & G2 CV & \%G2 & \%S & G2/G1 & \%Total & B.A.D. \\
\hline Diploid & 356.42 & 6.41 & 50.22 & 634.54 & 8.05 & 18.97 & 30.82 & 1.78 & 100.00 & 0.05 \\
\hline
\end{tabular}

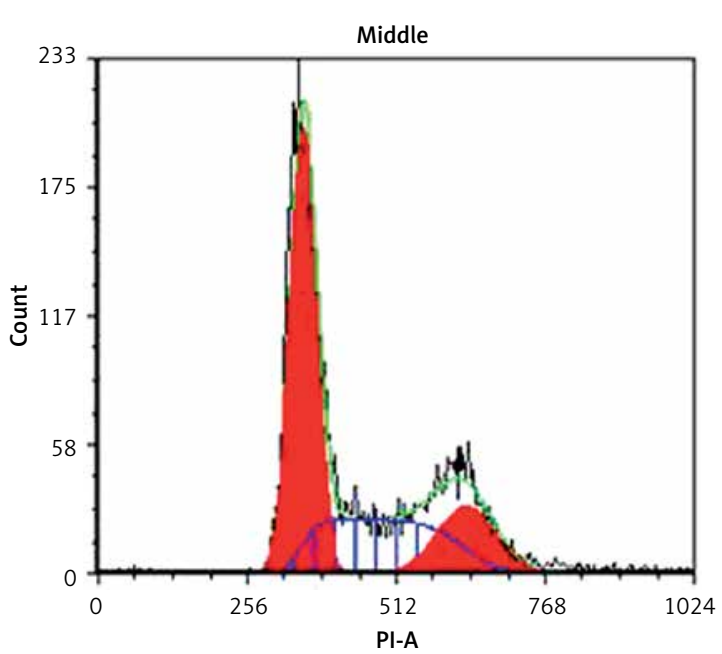

\begin{tabular}{|c|c|c|c|c|c|c|c|c|c|c|}
\hline Cycle & G1 Mean & G1 CV & \%G1 & G2 Mean & G2 CV & \%G2 & \%S & G2/G1 & \%Total & B.A.D. \\
\hline Diploid & 353.60 & 6.45 & 52.08 & 635.23 & 8.03 & 17.52 & 30.40 & 1.80 & 100.00 & 0.08 \\
\hline
\end{tabular}

Figure 1. Cont. C - The effect of EA on HeLa cell cycle progression was determined by flow cytometry: NC - control group. Low - EA low-dosage group ( $50 \mathrm{mg} / \mathrm{kg} / \mathrm{day}$ ). Middle - EA middle-dosage group (75 mg/kg/day). High - EA high-dosage group (100 mg/kg/day). 5-Fu - 5-Fu group. ${ }^{*} p<0.05$, compared with NC group; ${ }^{* \star} p<0.05$, compared with Low group; ${ }^{* * *} p<0.05$, compared with Middle group 


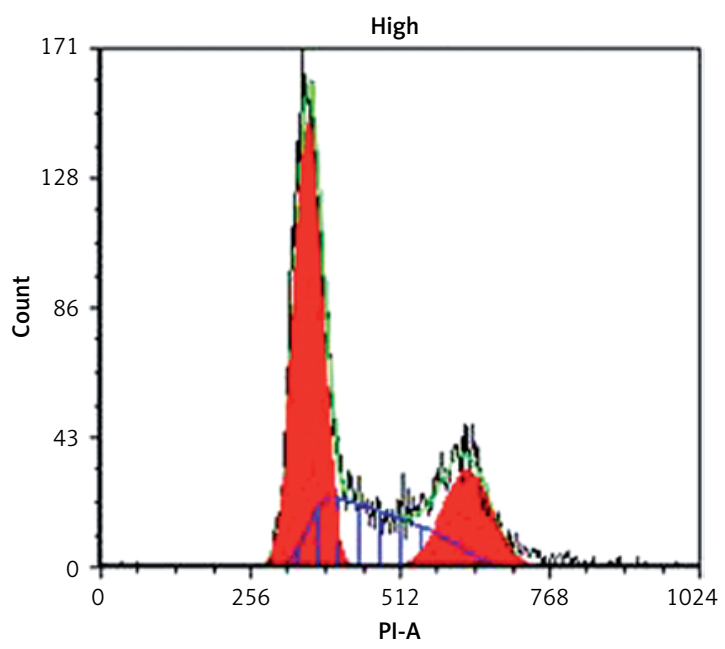

\begin{tabular}{|c|c|c|c|c|c|c|c|c|c|c|}
\hline Cycle & G1 Mean & G1 CV & \%G1 & G2 Mean & G2 CV & \%G2 & \%S & G2/G1 & \%Total & B.A.D. \\
\hline Diploid & 356.26 & 6.54 & 51.53 & 629.57 & 6.54 & 19.66 & 28.82 & 1.77 & 100.00 & 0.00 \\
\hline
\end{tabular}

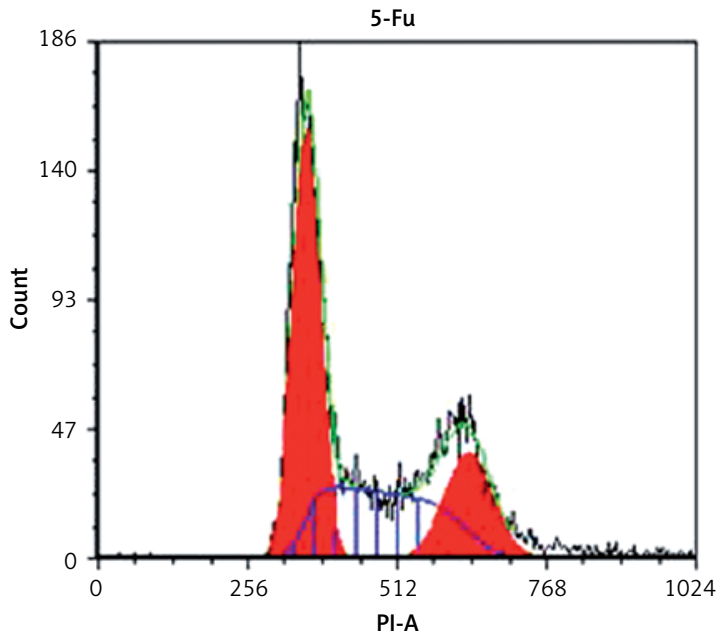

\begin{tabular}{|c|c|c|c|c|c|c|c|c|c|c|}
\hline Cycle & G1 Mean & G1 CV & \%G1 & G2 Mean & G2 CV & \%G2 & \%S & G2/G1 & \%Total & B.A.D. \\
\hline Diploid & 358.01 & 6.44 & 46.06 & 637.53 & 6.44 & 20.04 & 33.90 & 1.78 & 100.00 & 0.00 \\
\hline
\end{tabular}

Figure 1. Cont. C - The effect of EA on HeLa cell cycle progression was determined by flow cytometry: NC - control group. Low - EA low-dosage group ( $50 \mathrm{mg} / \mathrm{kg} /$ day). Middle - EA middle-dosage group $(75 \mathrm{mg} / \mathrm{kg} /$ day). High - EA high-dosage group (100 mg/kg/day). 5-Fu - 5-Fu group. ${ }^{*} p<0.05$, compared with NC group; ${ }^{* *} p<0.05$, compared with Low group; ${ }^{\star \star \star} p<0.05$, compared with Middle group 

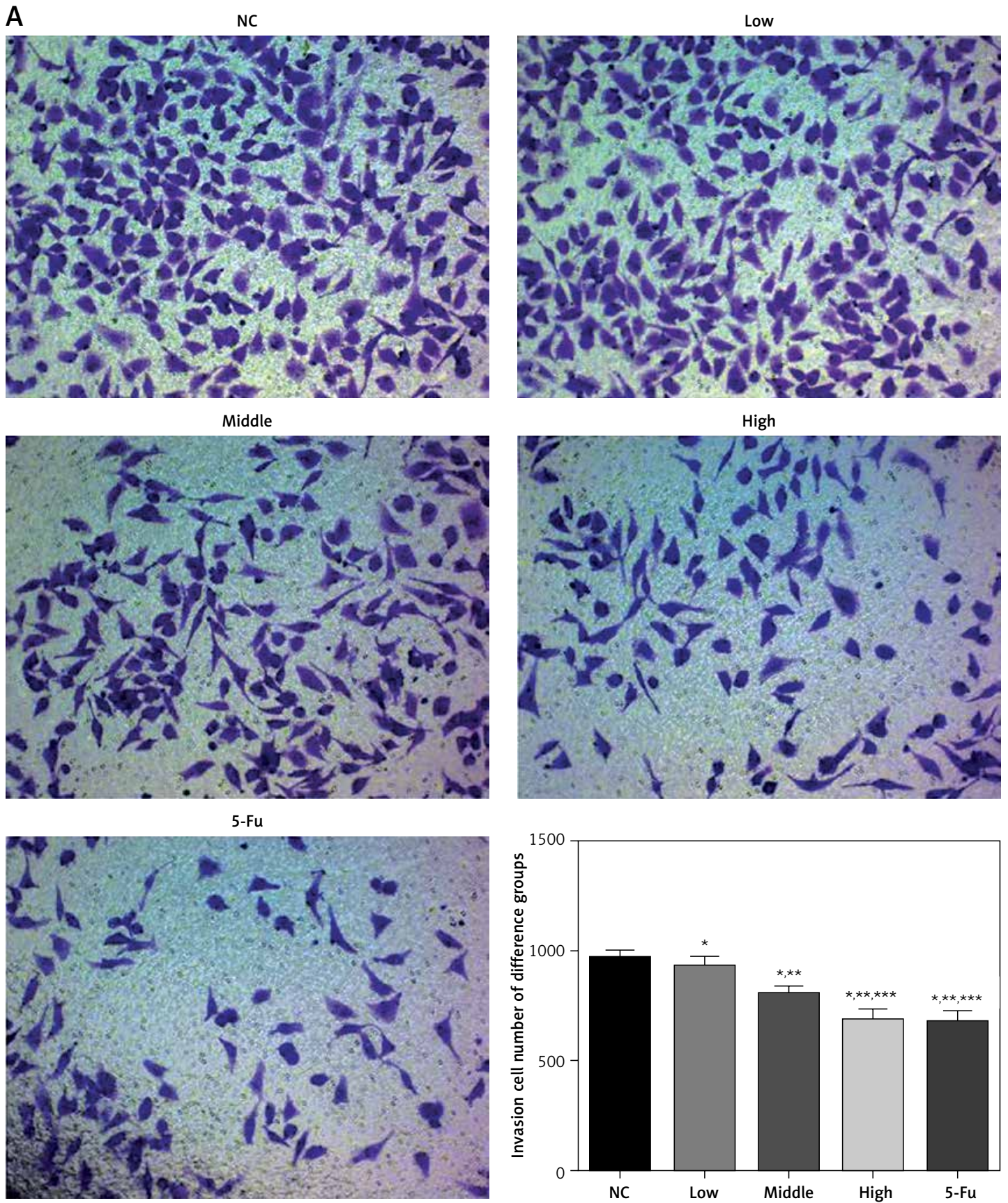

Figure 2. Effect of EA on HeLa cell invasion and migration. A - The effect of EA on HeLa cell invasion was determined by transwell assay 
B
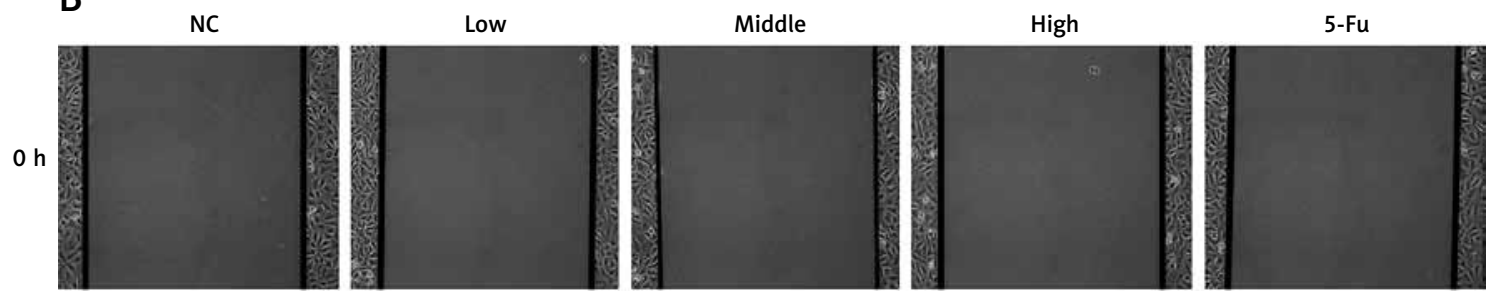

$24 \mathrm{~h}$
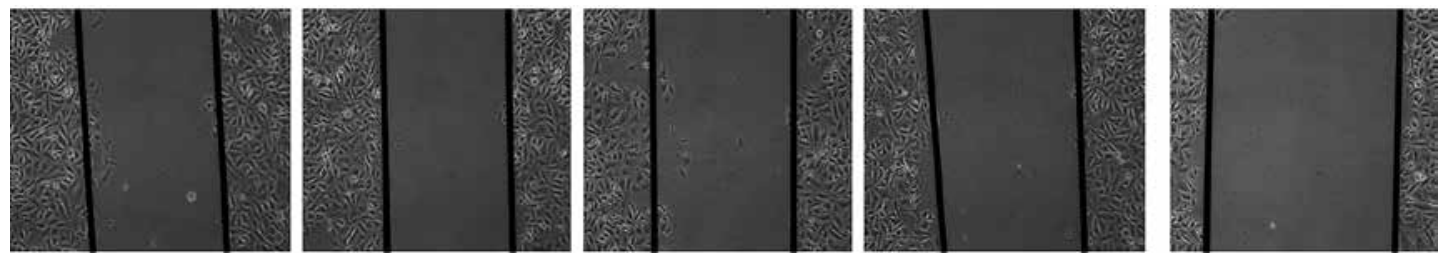

$48 \mathrm{~h}$
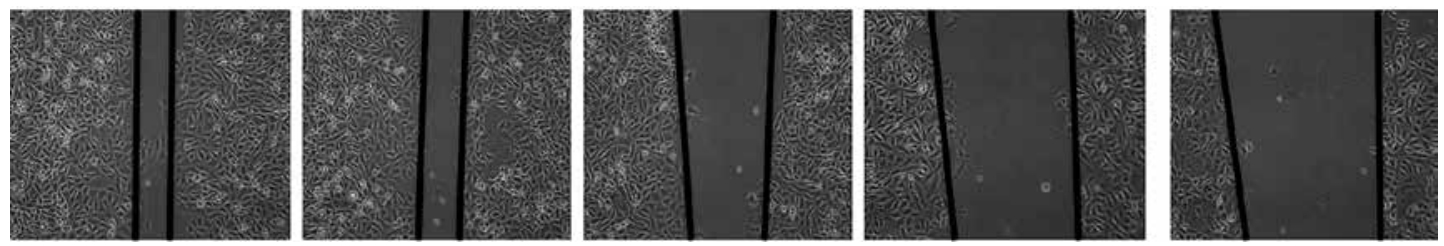

Figure 2. Cont. B - Effect of EA on HeLa cell migration was detected by wound closure assay. NC - cells treated with $0 \mu \mathrm{M}$ EA; Low - cells treated with $2.5 \mu \mathrm{M}$ EA; Middle - cells treated with $5 \mu \mathrm{M}$ EA; High - cells treated with $10 \mu M$ EA; 5 -Fu - cells treated with appropriate concentration of $5-\mathrm{Fu}$. ${ }^{*} p<0.05$, compared with NC group; ${ }^{* *} p<0.05$, compared with Low group; ${ }^{\star \star \star} p<0.05$, compared with Middle group 

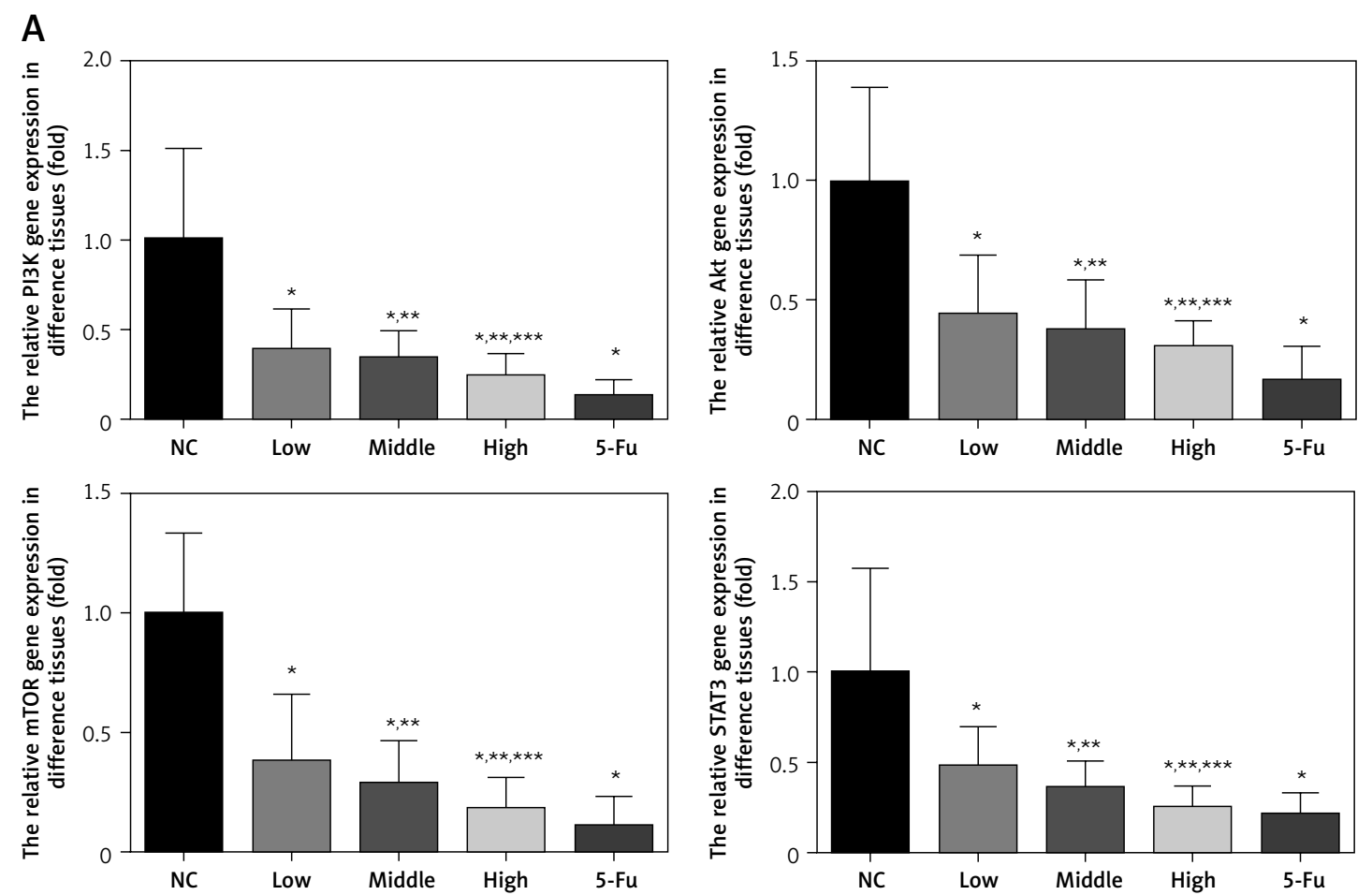

Figure 3. Effect of EA on activation of PI3K/AKT/mTOR/STAT3 signaling in HeLa cells. A - qRT-PCR was performed respectively to detect PI3K, AKT, mTOR and STAT3 gene expression 
B
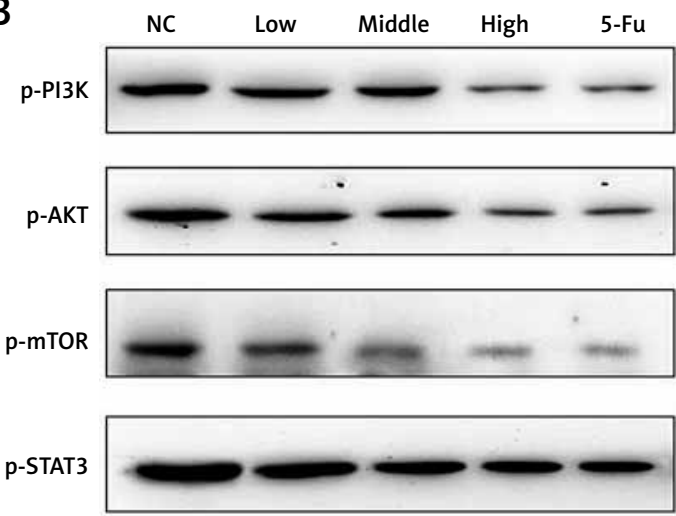

GAPDH
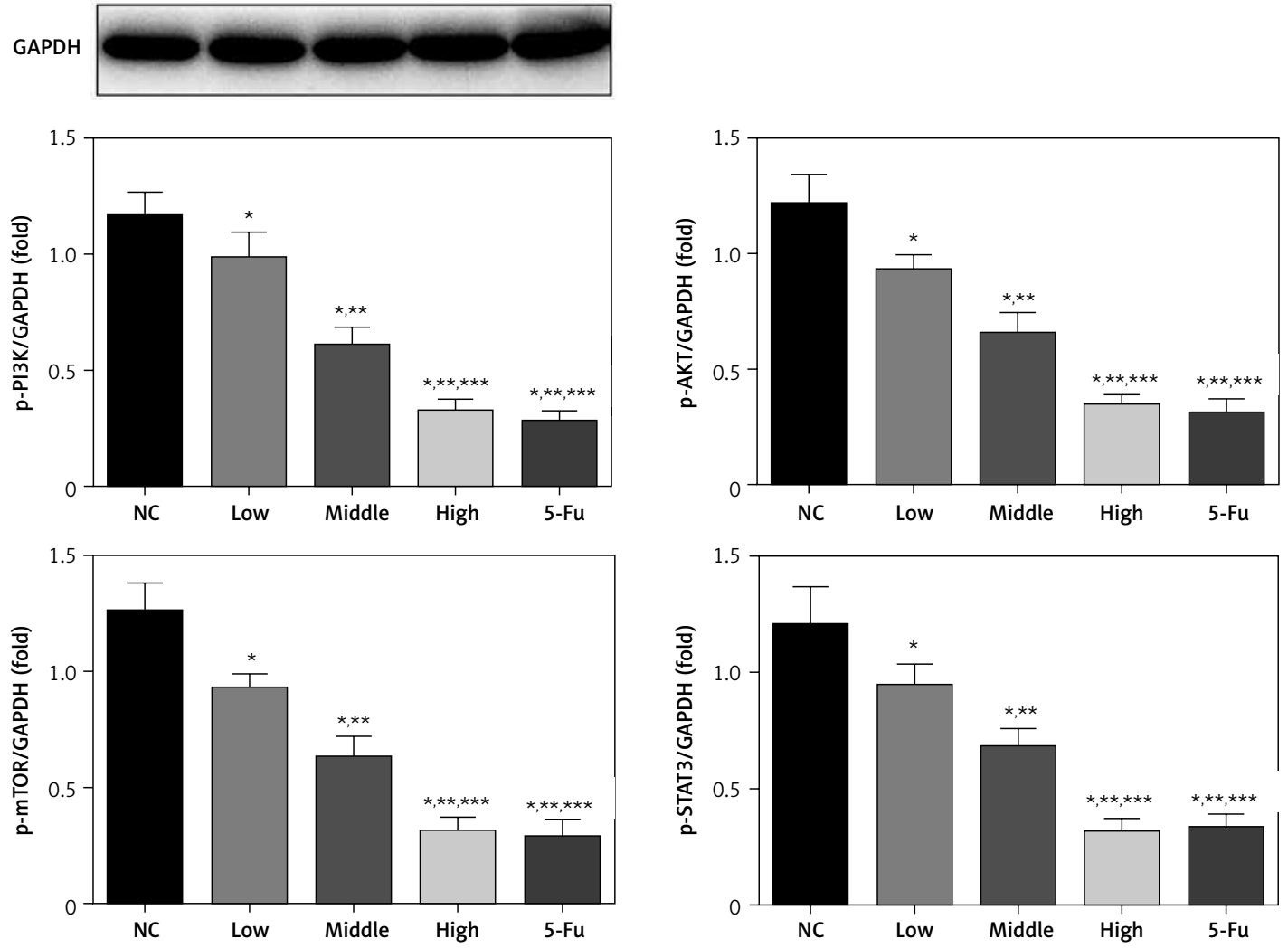

Figure 3. Cont. B - Western blotting was performed respectively to detect phospho-PI3K, phospho-AKT, phospho-mTOR and phospho-STAT3 protein expression. NC - cells treated with $0 \mu \mathrm{M}$ EA; Low - cells treated with $2.5 \mu \mathrm{M}$ EA; Middle - cells treated with $5 \mu$ M EA; High - cells treated with $10 \mu \mathrm{M}$ EA; 5 -Fu - cells treated with appropriate concentration of 5 -Fu. ${ }^{*} p<0.05$, compared with NC group; ${ }^{* *} p<0.05$, compared with Low group; ${ }^{* * *} p<0.05$, compared with Middle group 

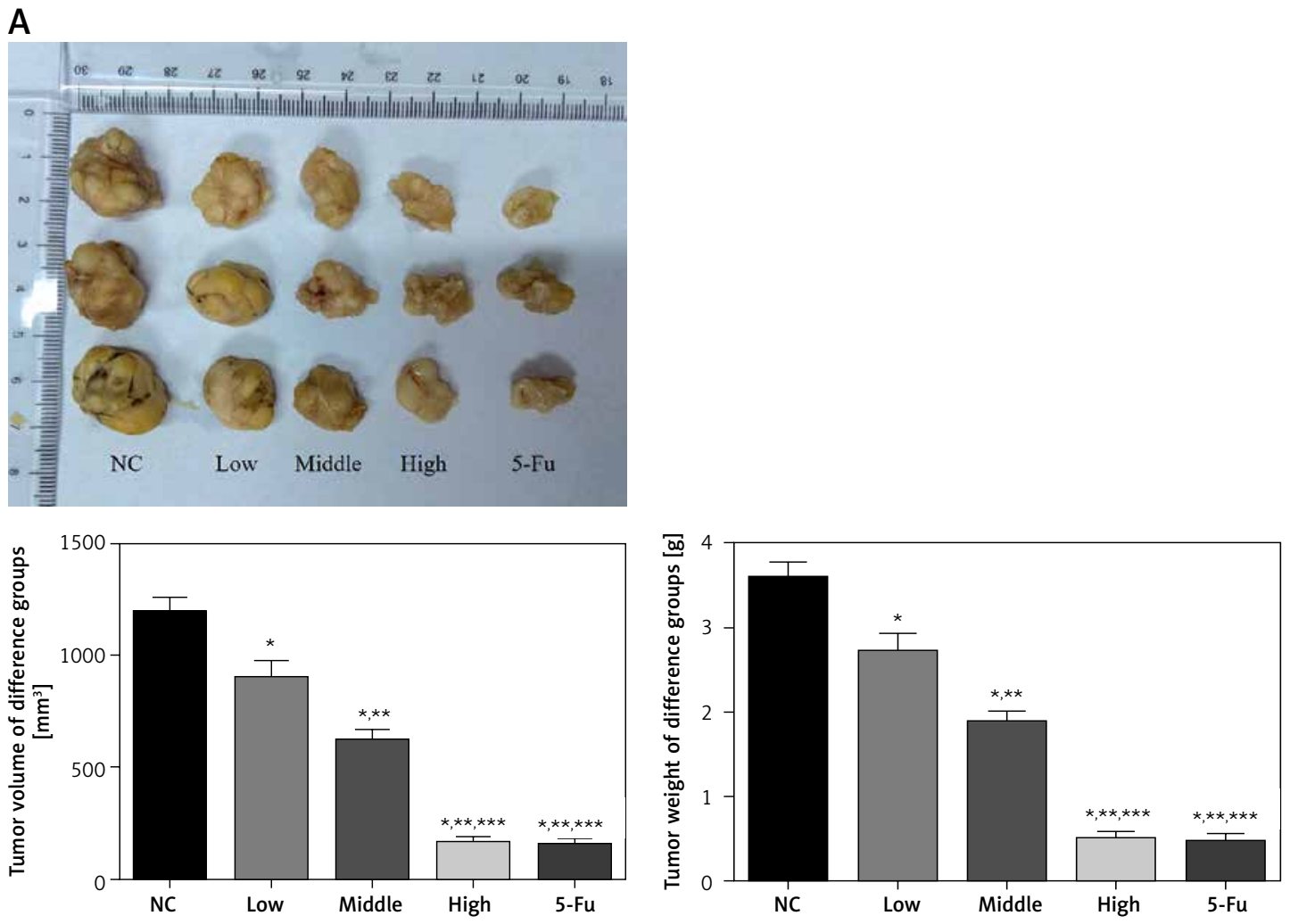

B

NC

Low
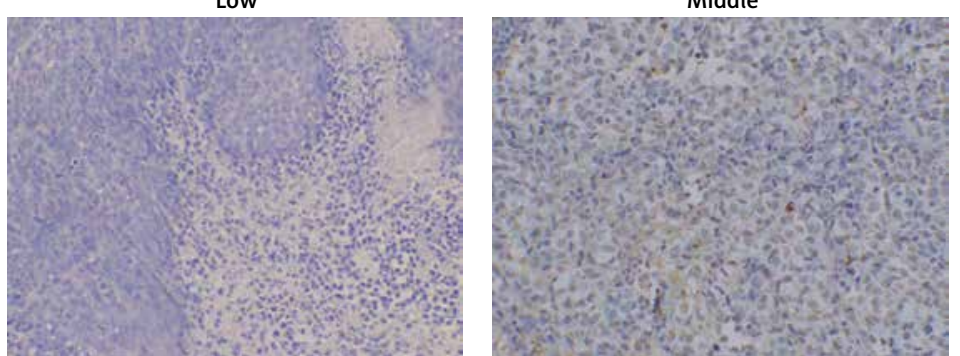

High

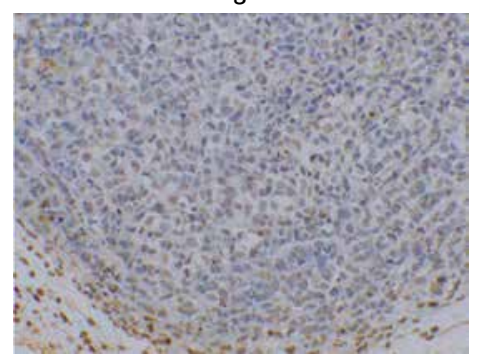

$5-\mathrm{Fu}$

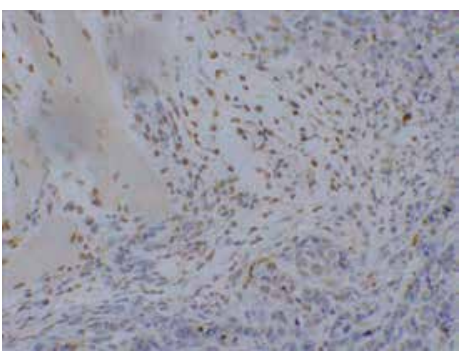

Figure 4. Effect of EA on cervical cancer growth in vivo. A - Nude mice bearing cervical tumor were treated daily with the vehicle or ellagic acid daily by intraperitoneal administration for 30 days. Representative tumor masses are shown. B - To test for apoptosis in EA-treated tumor tissues, TUNEL assay was performed. NC - cells treated with $0 \mu \mathrm{M}$ EA; Low - cells treated with $2.5 \mu \mathrm{M}$ EA; Middle - cells treated with $5 \mu \mathrm{M}$ EA; High - cells treated with $10 \mu \mathrm{M}$ EA; 5-Fu - cells treated with appropriate concentration of 5 -Fu. ${ }^{*} p<0.05$, compared with NC group; ${ }^{* *} p<$ 0.05 , compared with Low group; ${ }^{\star \star *} p<0.05$, compared with Middle group 
A
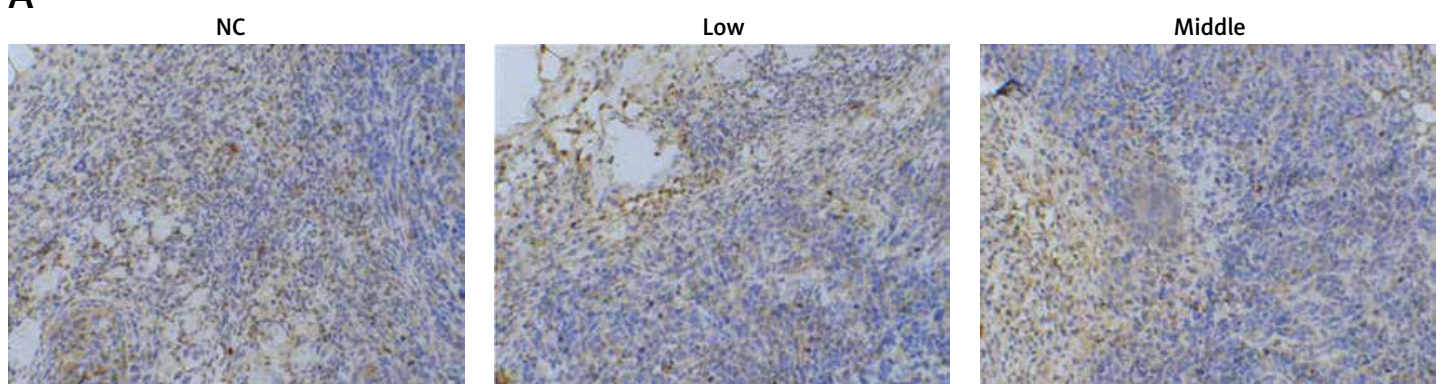

High

$5-\mathrm{Fu}$
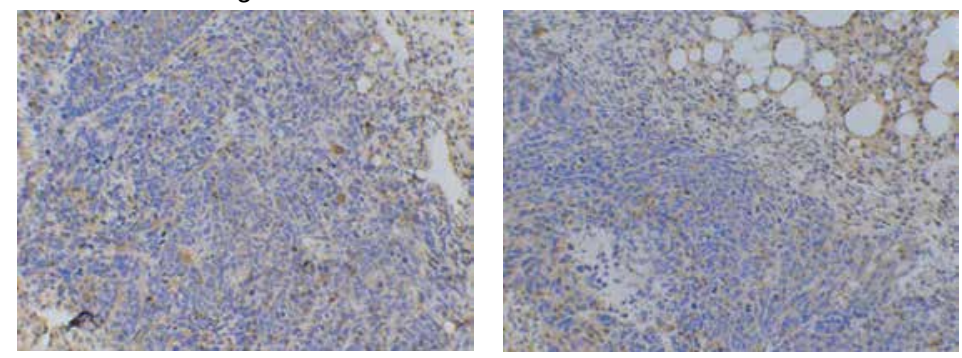

B
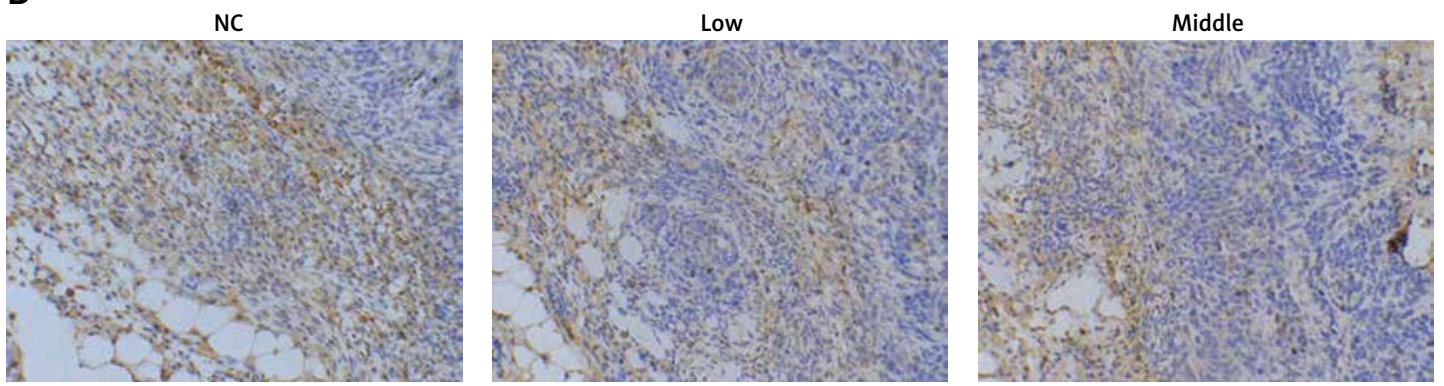

High

$5-\mathrm{Fu}$
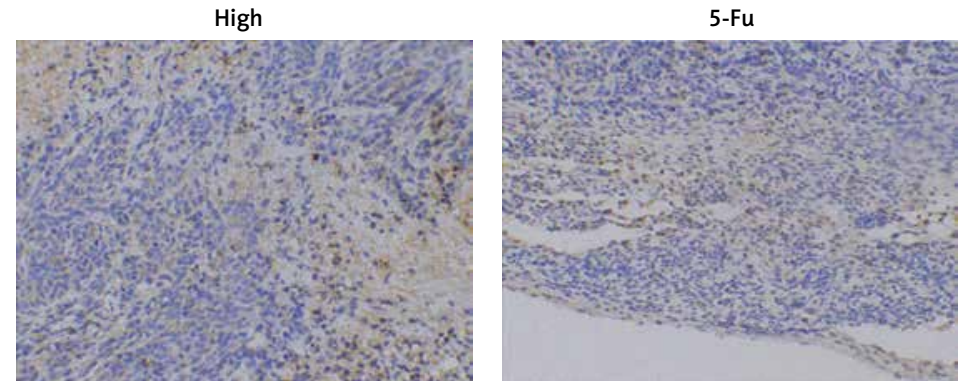

Figure 5. Relative protein expression in vivo. A-D - Tumor tissues were prepared for immunohistochemistry detection with specific antibodies against P-PI3K (A), P-AKT (B) 
C
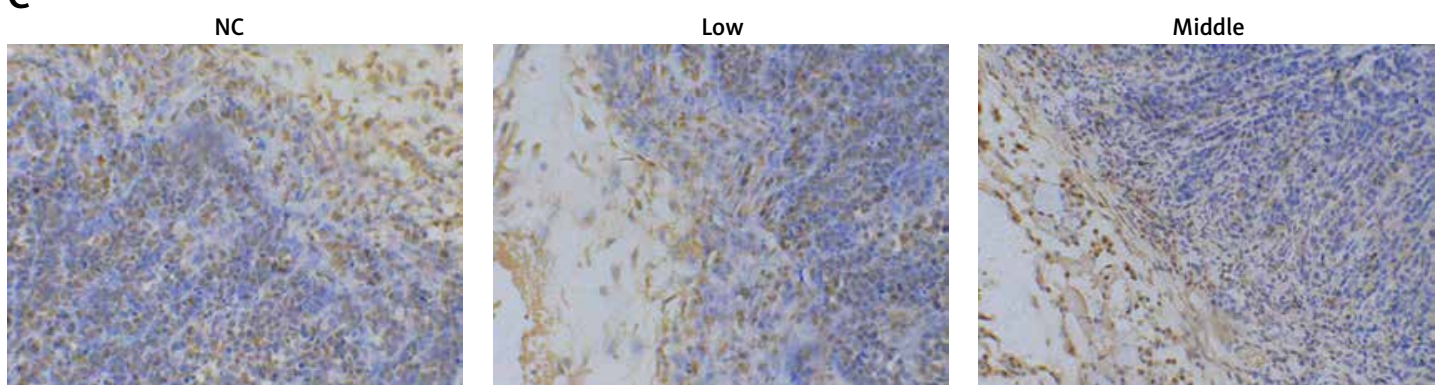

High

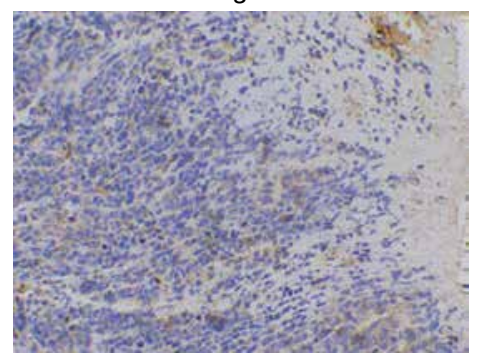

$5-\mathrm{Fu}$

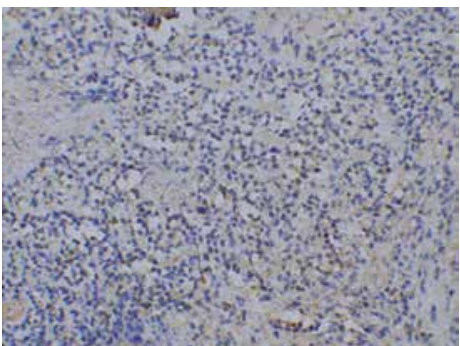

D
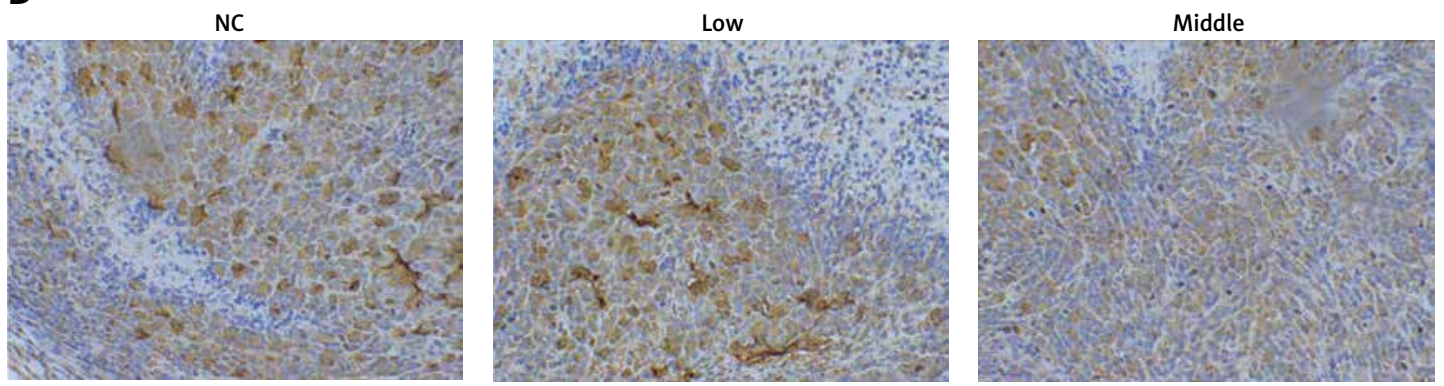

High
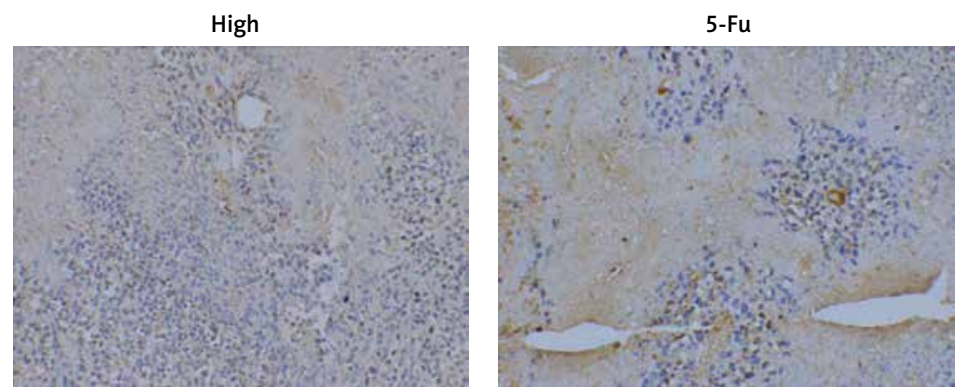

Figure 5. Cont. P-mTOR (C), and P-STAT3 (D). It was found that ellagic acid treatment could obviously attenuate expressions of P-PI3K, P-AKT, P-mTOR, and P-STAT3

Ellagic acid not only inhibited the growth and migration of HeLa cells, but also directly inhibited the growth of cervical cancer. EA inhibited the migration of HeLa cells. EA $100 \mathrm{mg} / \mathrm{kg} /$ day was injected intraperitoneally once a day. The results showed that EA had a significant inhibitory effect on the volume of cervical cancer, indicating that EA had a significant inhibitory effect on the growth of cervical cancer in vivo. At the same time, the results showed that EA could significantly inhibit the expression of $p$-PI3k, p-AKT, p-mTOR and $p$-STAT3 in tumor tissue. In general, our study shows that a non-toxic dose of EA shows strong anti-tumor growth activity by specifically targeting the AKT/mTOR/STAT3 signaling pathway.

\section{Conflict of interest}

The authors declare no conflict of interest.

\section{References}

1. Narayanan BA, Geoffroy O, Willingharm MC, et al. P53/ p21 (WAF1/CIP1) expression and its possible role in G1 arrest and apoptosis in Ellagic acid treated cancer cells. Cancer Lett 1999; 136: 215-21.

2. Qiu Z, Zhou B, Jin L, et al. In vitro antioxidant and antiproliferative effects of ellagic acid and its colonic me- 
tabolite, urolithins, on human bladder cancer T24 cells. Food Chem Toxicol 2013; 59: 428-37.

3. Larrosa M, Gonzalez-Sarras A, Yanez-Gascon MJ, et al. Anti-inflammatory properties of a pomegranate extract and its metabolite urolithin-A in a colitis rat model and the effect of colon inflammation on phenolic metabolism. J Nutr Biochem 2010; 21: 717-25.

4. Nandi S, Vracko M, Bagchi MC. Anticancer activity of selected phenolic compounds: QSAR studies using ridge regression and neural networks. Chem Biol Drug Des 2007; 70: 424-36.

5. Catherine RE, Nicholas M, George P. Antioxidant properties of phenolic compounds. Cell 1997; 2: 152-9.

6. Hayeshi R, Mutingwende I, Mavengere W, et al. The inhibition of human glutathione S-transferases activity by plant polyphenolic compounds ellagic acid and curcumin. Food Chem Toxicol 2007; 45: 286-95.

7. Larrosa M, Tomas-Barberan FA, Espin JC, et al. The dietary hydrolysable tannin punicalagin releases ellagic acid that induces apoptosis in human colon adenocarcinoma Caco- 2 cells by using the mitochondrial pathway. J Nutr Biochem 2006; 17: 611-25.

8. Mertens-Talcott SU, Jilma-Stohlawetz P, Rios J, et al. Absorption, metabolism, and antioxidant effects of pomegranate (Punica granatum l.) polyphenols after ingestion of a standardized extract in healthy human volunteers. J Agric Food Chem 2006; 54: 8956-61.

9. Thresiamma KC, Kuttan R. Inhibition of liver fibrosis by ellagic acid. Indian J Physiol Pharmacol 1996; 40: 363-6.

10. Jemal A, Bray F, Center MM, et al. Global cancer statistics. CA Cancer J Clin 2011; 61: 69-90.

11. Zur Hausen H. Papillomaviruses causing cancer: evasion from host-cell control in early events in carcinogenesis. J Natl Cancer Inst 2000; 92: 690-8.

12. Scheffner M, Huibregtse JM, Vierstra RD, et al. The HPV-16 E6 and E6-AP complex functions as a ubiquitin-protein ligase in the ubiquitination of p53. Cell 1993; 75: 495-505.

13. Kumar D, Basu S, Parija L, et al. Curcumin and Ellagic acid synergistically induce ROS generation, DNA damage, p53 accumulation and apoptosis in HeLa cervical carcinoma cells. Biomed Pharmacother 2016; 81: 31-7.

14. Chiarini F, Lonetti A, Teti G, et al. A combination of temsirolimus, an allosteric mTOR inhibitor, with clofarabine as a new therapeutic option for patients with acute myeloid leukemia. Oncotarget 2012; 3: 1615-28.

15. He SQ, Gao M, Fu YF, et al. Glycyrrhizic acid inhibits leukemia cell growth and migration via blocking AKT/mTOR/ STAT3 signaling. Int J Clin Exp Pathol 2015; 8: 5175-81.

16. Einaggar AC, Saini U, Naidu S, et al. Anticancer potential of diarylidenyl piperidone derivatives, $\mathrm{HO}-4200$ and $\mathrm{H}-4318$, in cisplatin resistant primary ovarian cancer. Cancer Biol Ther 2016; 17: 1107-15.

17. Seeram NP, Adams LS, Henning SM, et al. In vitro antiproliferative, apoptotic and antioxidant activities of punicalagin, ellagic acid and a total pomegranate tannin extract are enhanced in combination with other polyphenols as found in pomegranate juice. J Nutr Biochem 2005; 16: 360-7.

18. Malik A, Afaq S, Shahid M, et al. Influence of ellagic acid on prostate cancer cell proliferation: a caspase-dependent pathway. Asian Pac J Trop Med 2011; 4: 550-5.

19. Edderkaoui M, Odinokova I, Ohno I, et al. Ellagic acid induces apoptosis through inhibition of nuclear factor kappa B in pancreatic cancer cells. World J Gastroenterol 2008; 14: 3672-80.

20. Kumar D, Basu S, Panrija L, et al. Curcumin and ellagic acid synergistically induce ROS generation, DNA dam- age, p53 accumulation and apoptosis in HeLa cervical carcinoma cells. Biomed Pharmacother 2016; 81: 31-7.

21. Choi IJ, Cha MS, Park ES, et al. The efficacy of concurrent cisplatin and 5- flurouracil chemotherapy and radiation therapy for locally advanced cancer of the uterine cervix. J Gynecol Oncol 2008; 19: 129-34.

22. Ying C, Wang S, Lu Y, et al. Glucose fluctuation increased mesangial cell apoptosis related to AKT signal pathway. Arch Med Sci 2019; 15: 730-7.

23. Garg G, Shah JP, Toy EP. Comparison of surgery or radiotherapy on complications and quality of life in patients with the stage IB and IIA uterine cervical cancer. Gynecol Oncol 2010; 116: 587

24. Shukla S, Bharti AC, Hussain S, et al. Elimination of high-risk human papillomavirus type HPV16 infection by 'Praneem' polyherbal tablet in women with early cervical intraepithelial lesions. J Cancer Res Clin Oncol 2009; 135: 1701-9.

25. Oh PS, Lim KT. HeLa cells treated with phytoglycoprotein $(150 \mathrm{kDa})$ were killed by activation of caspase 3 via inhibitory activities of NF-kappaB and AP- 1. J Biomed Sci 2007; 14: 223-32.

26. Zhao S, Shao L, Wang Y, Meng Q, Yu J. Ketamine exhibits anti-gastric cancer activity via induction of apoptosis and attenuation of PI3K/Akt/mTOR. Arch Med Sci 2020; 16: 1140-9.

27. Sakamoto KM, Grant S, Saleiro D, et al. Target-ing novel signaling pathways for resistant acute myeloid leukemia. Mol Genet Metab 2015; 114: 397-402.

28. Wu CH, Chen AZ, Yen GC. Protective effects of glycyrrhizic acid and $18 \beta$-glycyrrhetinic acid against cisplatin-induced nephrotoxicity in BALB/c mice. J Agric Food Chem 2015; 63: 1200-9.

29. Jia X, Yang W, Han J, et al. Effects of lentivi-rus mediated STAT3 silencing on human chron-ic myeloid leukemia cells and leukemia mice. Int J Clin Exp Med 2014; 7: 4031-7.

30. Jordan NJ, Dutkowski CM, Barrow D, et al. Impact of dual mTORC1/2 mTOR ki-nase inhibitor AZD8055 on acquired endo-crine resistance in breast cancer in vitro. Breast Cancer Res 2014; 16: R12. 